\title{
Unintegrated Parton Distributions Functions - From Heavy Quarks to Nonphotonic Electrons
}

Marta Łuszczak*

University of Rzeszow, Poland

E-mail: luszczak@univ.rzeszow.pl

\section{Antoni Szczurek}

University of Rzeszow \& Institute of Nuclear Physics PAN, Poland

E-mail: Antoni.Szczurek@ifj.edu.pl

\begin{abstract}
We discuss production of nonphotonic electrons in proton-proton scattering at RHIC. We have calculated the inclusive cross section of heavy quarks, heavy mesons and electrons/positrons production in proton-proton and proton-antiproton collisions. The calculations are performed using an approach, based on the unintegrated parton distributions functions (UPDFs). In the first step we have calculated the single spectra of heavy quarks and antiquarks. In the next step to obtain the single particle spectra of mesons from those of quarks/antiquarks we used a standard hadronization procedure with Peterson and BCFY fragmentation functions. We find the semileptonic decay functions by fitting to recent data of the CLEO and BABAR collaborations. Finally we have calculated inclusive spectra of nonphotonic electrons/positrons for RHIC energy and compared with experimental data measured recently by the PHENIX collaboration at RHIC.
\end{abstract}

XXth Hadron Collider Physics Symposium

November 16 - 20, 2009

Evian, France

${ }^{*}$ Speaker. 


\section{Formalism}

Recently the PHENIX and STAR collaborations has measured transverse momentum distribution of nonphotonic electrons $[2,1]$. The whole procedure of electron/positron production can be written in the following schematic way:

$$
\frac{d \sigma^{e}}{d y d^{2} p}=\frac{d \sigma^{Q}}{d y d^{2} p} \otimes D_{Q \rightarrow D} \otimes f_{D \rightarrow e}
$$

where the symbol $\otimes$ denotes a generic convolution. The first term is responsible for production of heavy quarks/antiquarks. Next step is the process of formation of heavy mesons and the last ingredient describe semileptonic decays of heavy mesons to electrons/positrons.

- Heavy quarks production

The inclusive heavy quark/antiquark production we have calculated in the framework of the $k_{t}$-factorization. In this approach transverse momenta of initial partons are included and emission of gluons is encoded in so-called unintegrated gluon distributions (UGDFs).

In the leading-order (LO) approximation within the $k_{t}$-factorization approach the quadruply differential cross section in the rapidity of $Q\left(y_{1}\right)$, in the rapidity of $\bar{Q}\left(y_{2}\right)$ and the transverse momentum of $Q\left(p_{1}, t\right)$ and $Q\left(p_{2}, t\right)$ can be written as

$$
\begin{array}{r}
\frac{d \sigma}{d y_{1} d y_{2} d^{2} p_{1, t} d^{2} p_{2, t}}=\sum_{i, j} \int \frac{d^{2} \kappa_{1, t}}{\pi} \frac{d^{2} \kappa_{2, t}}{\pi} \frac{1}{16 \pi^{2}\left(x_{1} x_{2} s\right)^{2}} \overline{\left|\mathscr{M}_{i j}\right|^{2}} \\
\delta^{2}\left(\vec{\kappa}_{1, t}+\vec{\kappa}_{2, t}-\vec{p}_{1, t}-\vec{p}_{2, t}\right) \mathscr{F}_{i}\left(x_{1}, \kappa_{1, t}^{2}\right) \mathscr{F}_{j}\left(x_{2}, \kappa_{2, t}^{2}\right)
\end{array}
$$

where $\mathscr{F}_{i}\left(x_{1}, \kappa_{1, t}^{2}\right)$ and $\mathscr{F}_{j}\left(x_{2}, \kappa_{2, t}^{2}\right)$ are the so-called unintegrated gluon (parton) distributions. Now the unintegrated parton distributions must be evaluated at: $x_{1}=\frac{m_{1, t}}{\sqrt{s}} \exp \left(y_{1}\right)+\frac{m_{2, t}}{\sqrt{s}} \exp \left(y_{2}\right)$, $x_{2}=\frac{m_{1, t}}{\sqrt{s}} \exp \left(-y_{1}\right)+\frac{m_{2, t}}{\sqrt{s}} \exp \left(-y_{2}\right)$, where $m_{i, t}=\sqrt{p_{i, t}^{2}+m_{Q}^{2}}$.

- From quarks to mesons

The inclusive distributions of hadrons can be obtained through a convolution of inclusive distributions of heavy quarks/antiquarks and $\mathrm{Q} \rightarrow \mathrm{h}$ fragmentation functions

$$
\left.\frac{d \sigma\left(y_{h}, p_{t, h}\right)}{d y_{h} d^{2} p_{t, h}} \approx \int_{0}^{1} \frac{d z}{z^{2}} D_{Q \rightarrow h}(z) \frac{d \sigma_{g g \rightarrow Q}^{A}\left(y_{Q}, p_{t, Q}\right)}{d y_{Q} d^{2} p_{t, Q}}\right|_{\substack{y^{y}=y_{h} \\ p_{t, Q}=p_{t, h} / z}} .
$$

The hadronization of heavy quarks is usually done with the help of fragmentation functions. The Peterson fragmentation functions are often used in this context. Peterson fragmentation function

$$
D_{Q \rightarrow H}(z)=\frac{N}{z\left[1-(1 / z)-\varepsilon_{Q} /(1-z)\right]}
$$

where for the LO calculations $\varepsilon_{c}=0.05$ and $\varepsilon_{b}=0.006$ 
- Single leptons spectra

Two-dimensional distributions in rapidity and transverse momentum for charm quark/antiquark, D mesons and electrons/positrons.
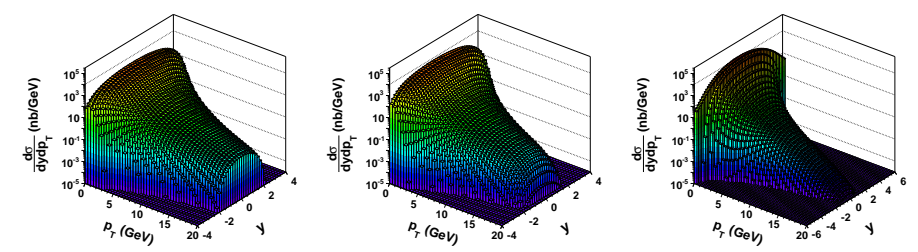

$p_{t}$ distributions of leptons with the Kwieciński UPDFs. Different combinations of factorization and renormalization scales are used.
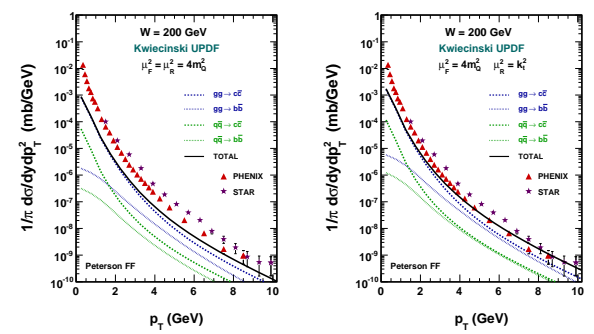

Factorization and renormalization uncertainty band (left) and quark mass uncertainty band (right) of our $k_{t}$-factorization calculation.
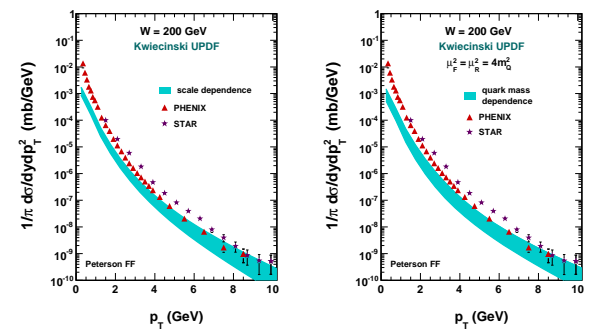

\section{Conclusions}

We have compared results obtained in our approach with experimental data measured recently by the PHENIX and STAR collaborations at RHIC. We get a reasonable description of the data at large transverse momenta of electrons/positrons. We have carefully studied uncertainties related to the choice of the factorization and renormalization scales, heavy quark masses and those related to the fragmentation process.

\section{References}

[1] J. Adams et al.(STAR collaboration), Phys. Rev. Lett. 94 (2005) 062301;

B.I. Abelev et al.(STAR collaboration), Phys. Rev. Lett. 98 (2007) 192301.

[2] A. Adare et al.(PHENIX collaboration), Phys. Rev. Lett. 97 (2006) 252002, arXiv:hep-ex/0609010. 